\title{
La familia Hemiaulaceae (Bacillariophyceae) de las aguas marinas chilenas
}

\author{
The family Hemiaulaceae (Bacillariophyceae) from marine Chilean waters \\ PATRICIO RIVERA ${ }^{1}$, SERGIO AVARIA $^{2} \&$ FABIOLA CRUCES $^{1}$ \\ ${ }^{1}$ Departamento de Botánica, Universidad de Concepción, Casilla 160-C, Concepción, Chile;
e-mail: privera@ udec.cl
${ }^{2}$ Facultad de Ciencias del Mar, Universidad de Valparaíso, Casilla 5080, Reñaca, Chile
}

\section{RESUMEN}

La familia Hemiaulaceae comprende cuatro géneros, señalados previamente para las aguas chilenas con las siguientes especies: Cerataulina pelagica (Cleve) Hendey, Hemiaulus sinensis Greville, H. membranaceus Cleve, Climacodium biconcavum Cleve, Eucampia antarctica (Castracane) Mangin, E. cornuta (Cleve) Grunow y E. zodiacus Ehrenberg. Sin embargo, el análisis con microscopía fotónica y electrónica de muestras recolectadas en aguas marinas chilenas que contenían representantes de esta familia (incluyendo la mayoría de aquellas estudiadas anteriormente por otros investigadores nacionales) reveló que (1) el género Cerataulina está representado por $C$. pelagica, distribuida a lo largo de la costa chilena entre Arica por el norte y el Estrecho de Magallanes por el sur, (2) el género Eucampia está representado por cuatro taxa: E. zodiacus f. cylindrocornis Syvertsen (señalada en trabajos anteriores como E. zodiacus f. zodiacus), E. zodiacus f. recta Rivera, Avaria \& Cruces f. nov. (descrita aquí), E. cornuta y E. antarctica. Los primeros tres taxa se distribuyen en la zona central y norte de Chile, mientras que E. antarctica es propia de las aguas antárticas desde el Estrecho de Magallanes al sur, (3) las citas anteriores de Hemiaulus sinensis, H. membranaceus y Climacodium biconcavum para las aguas chilenas corresponden a determinaciones erróneas de Eucampia zodiacus f. recta, f. nov. Se entregan descripciones de los taxa encontrados y fotografías obtenidas con los microscopios fotónico y electrónicos que ilustran sus principales características morfológicas.

Palabras clave: diatomeas, Hemiaulaceae, Chile, morfología, taxonomía.

\begin{abstract}
The Family Hemiaulaceae comprises four genera, all of which have been previously reported for coastal waters off Chile, and represented by the following taxa: Cerataulina pelagica (Cleve) Hendey, Hemiaulus sinensis Greville, H. membranaceus Cleve, Climacodium biconcavum Cleve, Eucampia antarctica (Castracane) Mangin, E. cornuta (Cleve) Grunow and E. zodiacus Ehrenberg. However, examination with light and electron microscopy of marine samples from along the Chilean coast and the Antarctic Peninsula (including those used in earlier publications) revealed that (1) the genus Cerataulina is represented by $C$. pelagica, and is distributed along the Chilean coast between Arica in the north and the Magellan Strait in the south, (2) the genus Eucampia comprises four taxa: E. zodiacus f. cylindrocornis Syvertsen (previously reported for Chile as E. zodiacus f. zodiacus), E. zodiacus f. recta Rivera, Avaria \& Cruces (described here), $E$. cornuta and E. antarctica. The former three taxa occur in the central and northern coast of Chile, while $E$. antarctica lives in Antarctic waters south of the Magellan Strait, (3) earlier records of Hemialus membranaceus, $H$. sinensis and Climacodium biconcavum in Chilean waters are misidentifications of Eucampia zodiacus f. recta f. nov. A short description is included for each taxon, and photographs provide information about the main morphological characteristics.
\end{abstract}

Key words: diatoms, Hemiaulaceae, Chile, morphology, taxonomy.

\section{INTRODUCCIÓN}

Los representantes de la familia Hemiaulaceae se caracterizan por (1) formar cadenas mediante la unión de una elevación que se presenta en cada extremo de las valvas, (2) poseer aréolas del tipo poroide, (3) tener un proceso labiado en cada valva y (4) sus numerosos cloroplastos de reducido tamaño (Hasle \& Syvertsen 1996). Comprende los géneros Cerataulina H. Peraga- 
llo ex Schütt, Hemiaulus Heiberg, Climacodium Grunow y Eucampia Ehrenberg. Se diferencian fundamentalmente por las características relacionadas con las elevaciones valvares (forma, largo), con los costate ocelli presentes en sus extremos y por la forma de sus cadenas (derechas, curvadas).

De acuerdo con Rivera (1983) y Rivera et al. (1990), todos estos géneros han sido señalados previamente para las aguas chilenas con las siguientes especies: Cerataulina pelagica (Cleve) Hendey, Hemiaulus sinensis Greville, $H$. membranaceus Cleve, Climacodium biconcavum Cleve, Eucampia antarctica (Castracane) Mangin, E. cornuta (Cleve) Grunow y E. zodiacus Ehrenberg. Exceptuando a E. antarctica que se distribuye en aguas antárticas hasta el Paso de Drake, las restantes especies son comunes en el fitoplancton marino nerítico de aguas chilenas, sin producir floraciones intensas.

En una serie de tiempo de treinta años en la Bahía de Valparaíso se han registrado en casi todos los años en concentraciones inferiores a 50.000 células $\mathrm{L}^{-1}$. En cruceros oceanográficos realizados en el norte de Chile, entre Arica y Antofagasta desde 1980 hasta 1984, se registraron también con bastante frecuencia, pero tan solo una vez se detectó, frente a Iquique, una floración de E. cornuta que alcanzó una concentración de 259.000 células $\mathrm{L}^{-1}$ (S. Avaria, resultados no publicados).

Los objetivos del presente trabajo son dar a conocer a la comunidad científica los géneros y especies pertenecientes a la familia Hemiaulaceae que están realmente presentes en las aguas marinas de Chile, describir la morfología de sus frústulos, las posibles diferencias existentes con poblaciones de otros lugares y actualizar su distribución a lo largo del área de estudio. Son muy pocos las investigaciones taxonómicas sobre diatomeas chilenas (marinas y de aguas dulces) que se llevan a cabo en nuestro país. Trabajos anteriores realizados por Rivera y colaboradores (e.g., Rivera 1981, 1985, Rivera \& Koch 1984, Rivera et al. 1996, 2002, 2003) han demostrado que nuestro conocimiento sobre esa flora es muy incompleto y que algunos taxa reportados para el país por otros autores han sido erróneamente identificados, originando con ello serios problemas para los fitoplanctólogos dedicados a la biogeografía o a la ecología de estas microalgas.

En el presente estudio se analizaron numerosas muestras de fitoplancton recolectadas en el Pacífico Suroriental que contenían representantes de esta familia, incluyendo la mayoría del material utilizado con anterioridad por otros investigadores nacionales que señalaron diversos taxa de la familia Hemiaulaceae para Chile.

Nuestros resultados revelan solamente la presencia en aguas chilenas de los géneros $\mathrm{Ce}$ rataulina y Eucampia. Las citas anteriores de los géneros Hemiaulus y Climacodium para la zona norte y central de Chile corresponden a determinaciones erróneas de Eucampia zodiacus f. recta Rivera, Avaria \& Cruces, f. nov., taxon que se describe por primera vez en este trabajo. Además, se señala por primera vez para Chile a E. zodiacus f. cylindrocornis Syvertsen, confundida anteriormente en Chile con la forma zodiacus Ehr., que no fue encontrada en el material analizado.

\section{MATERIALES Y MÉTODOS}

El material estudiado en el presente trabajo se encuentra depositado en la Colección Diatomológica de la Universidad de Concepción, Concepción, Chile (DIAT-CONC), y corresponde a muestras recolectadas a lo largo de la costa chilena y Antártica por diferentes expediciones y en distintos períodos (Tabla 1). Parte del material fue oxidado y montado en Hyrax siguiendo el método propuesto por Hasle \& Fryxell (1970). DIAT-CONC M- y un número, corresponde a una muestra líquida, mientras que DIAT-CONC y un número, corresponde a una preparación permanente. El microscopio fotónico utilizado fue un Zeiss Fotomicroscopio III. Las observaciones con microscopía electrónica se hicieron en un microscopio de barrido ETEC-Autoscan U-1 y en uno de transmisión JEOL-1200 Ex, pertenecientes al Laboratorio de Microscopía Electrónica de la Universidad de Concepción. Las medidas morfométricas de cada taxon se presentan en la Tabla 2. La terminología adoptada en este trabajo corresponde a la propuesta por Ross et al. (1979) y por Hasle \& Syvertsen (1996).

\section{RESULTADOS}

\section{Género Cerataulina H. Peragallo ex Schütt 1896}

Células cilíndricas, con numerosos cloroplastos, unidas en cadenas mediante una corta elevación que existe en cada uno de los polos de la valva y que lleva una prolongación aliforme de largo variable. Valvas circulares a subcirculares, presentando un proceso labiado distintamente ubicado según las especies. Cada una de las elevaciones valvares termina en un costate ocellus provisto de costillas paralelas. 


\section{TABLA 1}

Muestras analizadas. (*) Analizadas previamente por los autores señalados

Samples examined. (*) Previously examined by the cited authors

\begin{tabular}{|c|c|c|c|}
\hline Número & Fecha & Localización & Observaciones \\
\hline DIAT-CONC M- 773 & 18 Agosto 1972 & $\begin{array}{l}\text { Mar Chile } \\
18^{\circ} 20^{\prime} \mathrm{S}, 74^{\circ} 16^{\prime} \mathrm{O}\end{array}$ & \\
\hline DIAT-CONC M- 3199 & Мayo 1981 & $\begin{array}{l}\text { Bahía de Iquique } \\
\text { Aprox. } 20^{\circ} 12^{\prime} \mathrm{S}, 70^{\circ} 10^{\prime} \mathrm{O}\end{array}$ & Colector: S. Avaria \\
\hline DIAT-CONC M- 853 & 10 Diciembre 1963 & $\begin{array}{l}\text { Bahía de Valparaíso } \\
33^{\circ} 02^{\prime} \mathrm{S}, 71^{\circ} 38^{\prime} \mathrm{O}\end{array}$ & Colector: S. Avaria \\
\hline DIAT-CONC M- 2433 & 1977 & $\begin{array}{l}\text { Bahía de Valparaíso } \\
33^{\circ} 02^{\prime} \mathrm{S}, 71^{\circ} 38^{\prime} \mathrm{O}\end{array}$ & Colector: S. Avaria \\
\hline DIAT-CONC M- 2419 & 24 Octubre 1977 & $\begin{array}{l}\text { Bahía de Valparaíso } \\
33^{\circ} 02^{\prime} \mathrm{S}, 71^{\circ} 38^{\prime} \mathrm{O}\end{array}$ & $\begin{array}{l}\text { Colector: S. Avaria } \\
\text { *Alvial \& Avaria (1982) }\end{array}$ \\
\hline DIAT-CONC M- 2420 & 4 Julio 1979 & $\begin{array}{l}\text { Bahía de Valparaíso } \\
33^{\circ} 02^{\prime} \mathrm{S}, 71^{\circ} 38^{\prime} \mathrm{O}\end{array}$ & $\begin{array}{l}\text { Colector: S. Avaria } \\
\text { *Avaria \& Muñoz (1982) }\end{array}$ \\
\hline DIAT-CONC M- 3189 & 11 Noviembre 1998 & $\begin{array}{l}\text { Bahía de Valparaíso } \\
33^{\circ} 02^{\prime} \mathrm{S}, 71^{\circ} 38^{\prime} \mathrm{O}\end{array}$ & Colector: S. Avaria \\
\hline DIAT-CONC M- 3188 & 4 Enero 1989 & $\begin{array}{l}\text { Bahía de Valparaíso } \\
33^{\circ} 02^{\prime} \mathrm{S}, 71^{\circ} 38^{\prime} \mathrm{O}\end{array}$ & Colector: S. Avaria \\
\hline DIAT-CONC M- 2093 & 9 Febrero 1985 & $\begin{array}{l}\text { Bahía de Coliumo } \\
36^{\circ} 32^{\prime} \mathrm{S}, 72^{\circ} 57^{\prime} \mathrm{O}\end{array}$ & \\
\hline DIAT-CONC M- 2263 & 23 Abril 1966 & $\begin{array}{l}\text { Bahía de Concepción, } \\
\text { Caleta Leandro, Tumbes } \\
36^{\circ} 40^{\prime} \mathrm{S}, 73^{\circ} 07^{\prime} \mathrm{O}\end{array}$ & $\begin{array}{l}\text { Colector: J. Hermosilla } \\
\text { *Rivera (1969) }\end{array}$ \\
\hline DIAT-CONC M- 207 & 15 Octubre 1970 & $\begin{array}{l}\text { Bahía de Concepción } \\
36^{\circ} 42^{\prime} \mathrm{S}, 73^{\circ} 02^{\prime} \mathrm{O}\end{array}$ & Colector: D. Arcos \\
\hline DIAT-CONC M- 871 & 4 Diciembre 1965 & $\begin{array}{l}\text { Bahía de Concepción } \\
36^{\circ} 42^{\prime} \mathrm{S}, 73^{\circ} 02^{\prime} \mathrm{O}\end{array}$ & $\begin{array}{l}\text { Colector: P. Rivera } \\
\text { *Rivera }(1969)\end{array}$ \\
\hline DIAT-CONC M- 1054 & 21 Noviembre 1965 & $\begin{array}{l}\text { Bahía de Concepción } \\
36^{\circ} 42^{\prime} \mathrm{S}, 73^{\circ} 02^{\prime} \mathrm{O}\end{array}$ & $\begin{array}{l}\text { Col. P. Rivera } \\
\text { *Rivera (1969) }\end{array}$ \\
\hline DIAT-CONC M- 2199 & 4 Diciembre 1965 & $\begin{array}{l}\text { Bahía de Concepción, El Morro, } \\
\text { Talcahuano, } 36^{\circ} 42^{\prime} \mathrm{S}, 73^{\circ} 02^{\prime} \mathrm{O}\end{array}$ & $\begin{array}{l}\text { Colector: J. Hermosilla } \\
\text { *Rivera }(1969)\end{array}$ \\
\hline DIAT-CONC M- 4 & 15 Octubre 1970 & $\begin{array}{l}\text { Estero Lenga } \\
36^{\circ} 46^{\prime} \mathrm{S}, 73^{\circ} 11^{\prime} \mathrm{O}\end{array}$ & $\begin{array}{l}\text { Colector: P. Rivera } \\
\text { *Rivera et al. (1973) }\end{array}$ \\
\hline DIAT-CONC M- 1356 & 22 Noviembre 1978 & $38^{\circ} 00^{\prime} \mathrm{S}, 73^{\circ} 50^{\prime} \mathrm{O}$ & Colector: P. Rivera \\
\hline DIAT-CONC 2734 & Diciembre 1982 & $\begin{array}{l}\text { Bahía de Corral } \\
39^{\circ} 52^{\prime} \mathrm{S}, 73^{\circ} 25^{\prime} \mathrm{O}\end{array}$ & Colector: J.C. Uribe \\
\hline DIAT-CONC M- 459 & 15 Enero 1972 & $\begin{array}{l}\text { Bahía Foster } \\
62^{\circ} 58^{\prime} 2^{\prime} \text { ' S, } 60^{\circ} 42^{\prime} 1^{\prime}, \mathrm{O}\end{array}$ & Antártica \\
\hline DIAT-CONC M- 524 & 19 Enero 1973 & $\begin{array}{l}\text { Bahía Andvord } \\
64^{\circ} 51^{\prime} 00^{\prime}, \mathrm{S}, 62^{\circ} 37^{\prime} 09^{\prime \prime} \mathrm{O}\end{array}$ & Antártica \\
\hline
\end{tabular}


Cerataulina pelagica (Cleve) Hendey, p. 279 (1937), Fig. 1.

Referencias: Hasle \& Syvertsen (1980, 1996), Takano (1990).

Material estudiado: DIAT-CONC M- 4, 207, 853, 2199, 2734.

Células cilíndricas, $12-48 \mu \mathrm{m}$ de diámetro y 23-60 $\mu \mathrm{m}$ de largo, unidas en cadenas levemente giradas respecto al eje pervalvar, dejando una separación muy pequeña entre dos valvas adyacentes (Fig. 1A, 1B). Cingulum formado por bandas abiertas, iguales entre sí, provistas de lígula y antilígula en posición opuesta (Fig. 1K), con líneas verticales de poros, 4-6 en $1 \mu \mathrm{m}$ (Fig. 1L). Un área no perforada se presenta en el lado advalvar y a lo largo de todas las bandas (Fig. 1K, 1L). Valvas circulares a subcirculares, llevando un proceso labiado en posición central o subcentral (Fig. 1C, 1D, 1F, 1H). Proceso labiado alargado, rodeado de un área hialina no perforada, orientado hacia los costate ocelli (Fig. 1E, 1H, 1I) o perpendicular a esa dirección (Fig. 1D, 1F) y levemente elevado sobre la cara externa de la valva (Fig. 1F, 1I). Aréolas de contorno cuadrangular (Fig. 1J), formando estrías orientadas radialmente hacia el proceso labiado (Fig. 1H, 1I), 17-26 en 10 $\mu \mathrm{m}$ sobre la cara valvar y algo más numerosas, 30-33 en $10 \mu \mathrm{m}$ en el manto valvar. Cada costate ocellus está provisto de una corta expansión aliforme, que se introduce en una depresión existente en la valva adyacente (Fig. $1 \mathrm{E}-1 \mathrm{G}, 1 \mathrm{I})$.

Distribución: especie cosmopolita, señalada para la costa chilena entre Arica $\left(18^{\circ} 28^{\prime} \mathrm{S}\right)$ y el Estrecho de Magallanes (aproximadamente 5300' S) (Rivera 1983, Rivera et al. 1990, Vera et al. 1996, Avaria et al. 1997).

\section{Género Eucampia Ehrenberg 1841}

Células con numerosos cloroplastos, unidas en cadenas mediante una elevación de la valva que se encuentra en cada uno de sus extremos; cadenas rectas o helicoidalmente curvadas en vista conectival ancha. Elevaciones valvares largas o cortas, truncadas, dejando siempre una notoria separación entre dos valvas adyacentes, y llevando en su extremo un costate ocellus con las costillas orientadas en forma radial, paralelas o paralelas al centro y radiales en los extremos. Valvas lanceoladas a elípticas, con un proceso labiado de posición central o marginal según las especies, no prolongado hacia el exterior. En el Anexo 1 se entrega una clave para separar las especies de Eucampia encontradas en el material estudiado.
Eucampia antarctica (Castracane) Mangin p. 480 (1914), Fig. 2.

Sinónimo: Eucampia balaustium Castracane, p. 97 (1886).

Referencias: Ross et al. (1977), Syvertsen \& Hasle (1983), Fryxell (1989), Fryxell et al. (1989), Fryxell \& Prasad (1990), Hasle \& Syvertsen (1996).

Material estudiado: DIAT-CONC M- 459, 524.

Aunque la mayor parte del material analizado estaba constituido por células aisladas, también fueron observas algunas cadenas cortas de 2-3 células, derechas en vista conectival ancha (Fig. 2A), pero levemente arqueadas en vista conectival angosta. Cada cingulum está formado por 5-12 bandas abiertas provistas de líneas de poros un tanto alargados (Fig. 2F, $2 \mathrm{G}), 24-33$ en $10 \mu \mathrm{m}$; un área no perforada se presenta en el lado advalvar de cada banda, separando los primeros 4-5 poros de cada línea (Fig. 2F, 2I). Valvas elípticas (Fig. 2B, 2C), 36-90 $\mu \mathrm{m}$ de largo, con elevaciones valvares relativamente anchas, truncadas (Fig. 2A), cada una provista de un costate ocellus con costillas paralelas, rodeado de un área hialina no siempre bien nítida (Fig. 2H, 2K). En vista conectival ancha, las valvas son planas o levemente convexas. Un proceso labiado, carente de prolongación externa, se sitúa 2-3 aréolas separado del margen valvar (Fig. 2C2E). Aréolas rectangulares y/o angulares (Fig. $2 \mathrm{C}, 2 \mathrm{H}, 2 \mathrm{~J}, 2 \mathrm{~K}), 5-9$ en $10 \mu \mathrm{m}$, orientadas hacia el proceso labiado.

Distribución: taxón propio de aguas antárticas, señalada por Meyer (1966) para el Paso de Drake.

Eucampia zodiacus Ehrenberg f. cylindrocornis Syvertsen, p. 173 (1983). Fig. 3.

Referencias: Syvertsen \& Hasle (1983), Hasle \& Syvertsen (1996).

Material estudiado: DIAT-CONC M- 773, 871, 2199, 2734.

Células cilíndricas, unidas en cadenas curvadas en vista conectival ancha (Fig. 3A). Cingulum formado por numerosas bandas abiertas, provistas de líneas paralelas de poros redondeados, 35-60 en $10 \mu \mathrm{m}$; un área no perforada se presenta en la parte central de cada banda (Fig. 3F). Valvas elípticas, eje apical 23-37 $\mu \mathrm{m}$, con elevaciones relativamente largas $(7-9 \mu \mathrm{m})$, subcilíndricas (Fig. 3B, 3C); cara valvar levemente cóncava en vista conectival ancha, dejando aberturas más o menos rectangulares entre las células (Fig. 3C). Aréolas 10-14 en $10 \mu \mathrm{m}$, cuadrangulares a rectangulares, dispuestas en líneas radiales a partir de un proceso labiado central carente de prolongación externa (Fig. 3D). Costate ocellus ovalado (Fig. 3D) y pro- 


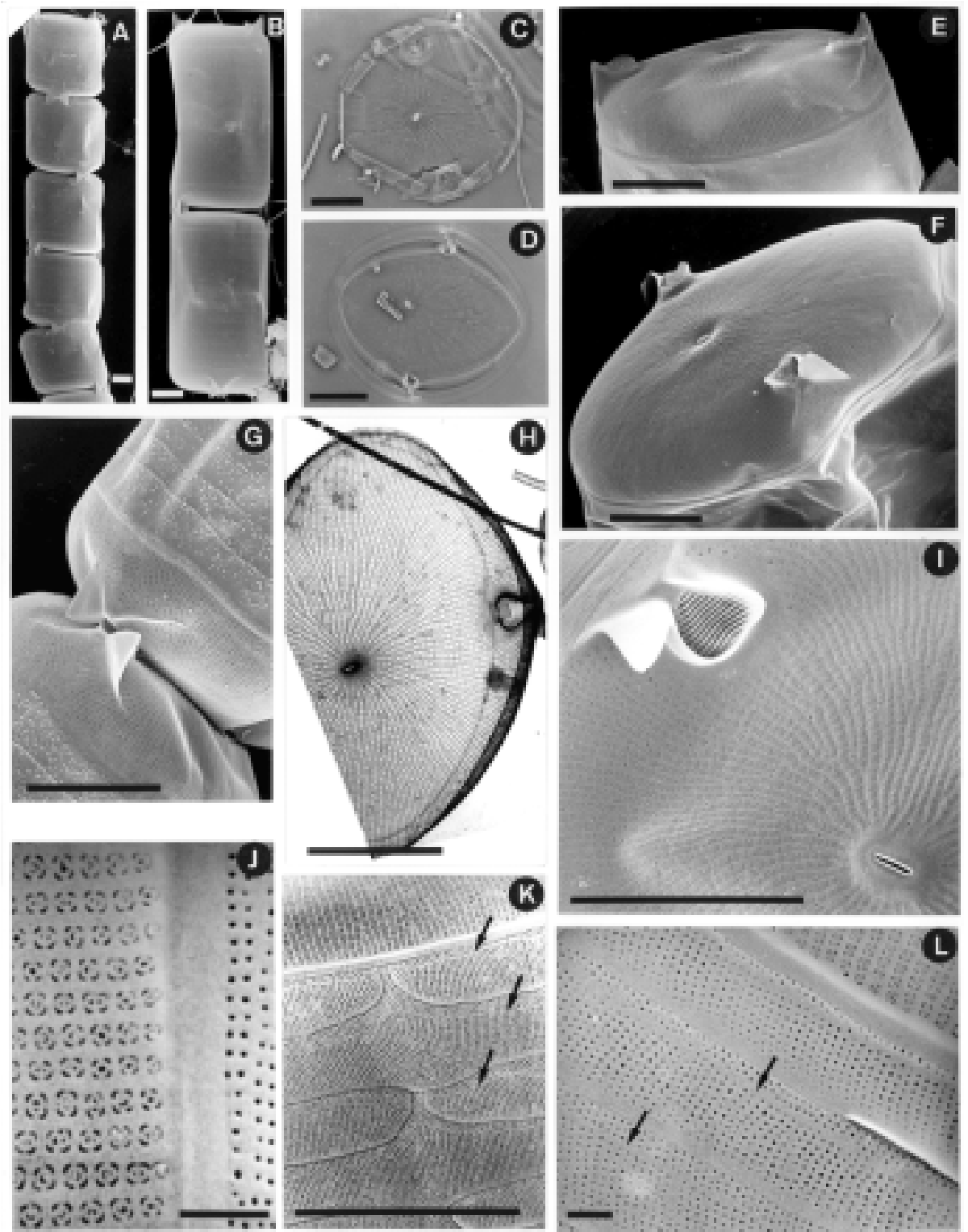

Fig. 1: Cerataulina pelagica (Cleve) Hendey. (A, B, E-G, I-L) MEB; (C, D) ML; (H) MET. Escala: $10 \mu \mathrm{m}(\mathrm{A}-\mathrm{I}, \mathrm{K}), 1 \mu \mathrm{m}(\mathrm{J}, \mathrm{L})$. (A) cadena de 6 células, note la torsión de las tecas; (B) dos células adyacentes de una cadena con una pequeña separación entre las dos valvas; $(C, D)$ contorno valvar, proceso labiado subcentral, procesos marginales; (E, F) disposición de las aréolas, costate ocelli marginales y orientación diferente del proceso labiado; $(\mathrm{G})$ dos valvas adyacentes y estructuras aliformes de los costate ocelli; $(\mathrm{H})$ vista interna de una valva, proceso labiado orientado hacia el costate ocellus y aréolas radiales; (I) costate ocellus con expansión aliforme y costillas paralelas, vista exterior del proceso labiado; ( $\mathrm{J}$ ) margen valvar y primera banda del cingulum, note la forma cuadrangular de las aréolas y la cribra; $(\mathrm{K}, \mathrm{L})$ parte del cingulum, bandas abiertas con lígula (arriba) y antilígula (abajo), note área no perforada por poroides hacia el lado advalvar (flechas).

Cerataulina pelagica (Cleve) Hendey. (A, B, E-G, I-L) SEM; (C, D) LM; (H) TEM. Scale: $10 \mu \mathrm{m}(\mathrm{A}-\mathrm{I}, \mathrm{K}), 1 \mu \mathrm{m}(\mathrm{J}, \mathrm{L})$. (A) chain of six cells, note the torsion of the thecas; (B) two adjacent cells of a chain with a short distance between the two valves; (C, D) shape of valves, subcentral labiate process, marginal processes; (E, F) areolae array, marginal costate ocelli, and orientation of labiate process; $(\mathrm{G})$ two adjacent valves and costate ocelli with wing-like prolongation; (H) internal view of a single valve, labiate process oriented to the costate ocellus and radial areolae; (I) costate ocellus with a wing-like prolongation and parallel costae, external view of the labiate process; ( $\mathrm{J}$ ) valve margin and first band of the cingulum, notice the square shape of the areolae and the cribra; $(\mathrm{K}, \mathrm{L})$ part of a cingulum, open bands with ligula (top) and antiligula (bottom), note a non- perforated area toward the advalvar side of each band (arrows). 

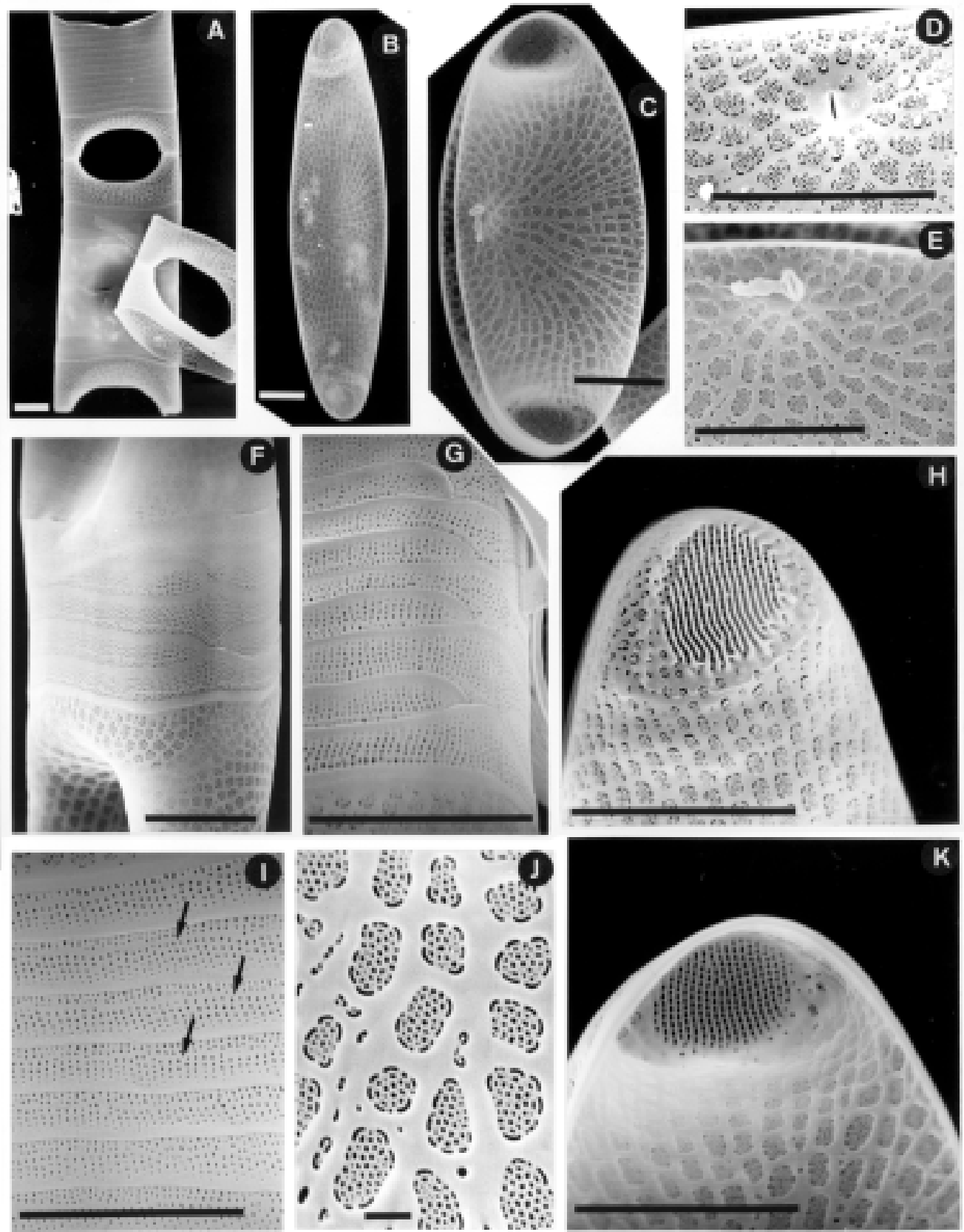

Fig. 2: Eucampia antarctica (Castracane) Mangin. MEB. Escala: $10 \mu \mathrm{m}$ (A-I, K), $1 \mu \mathrm{m}(\mathrm{J})$. (A) cadena derecha, corta, vista conectival ancha; (B, C) valvas en vista externa e interna, aréolas orientadas hacia el proceso labiado marginal; (D) abertura externa del proceso labiado; (E) proceso labiado, vista interna, separado 2-3 aréolas del margen valvar; $(F, G)$ bandas abiertas del cingulum, con lígula y antilígula; (H) vista externa del costate ocellus, anillo hialino no bien definido; (I) parte del cingulum, note los poros alargados de las bandas y el área no perforada hacia el lado advalvar (flechas); (J) aréolas sobre la cara valvar; (K) vista interna del costate ocellus provisto de un anillo hialino.

Eucampia antarctica (Castracane) Mangin. SEM. Scale: $10 \mu \mathrm{m}$ (A-I, K), $1 \mu \mathrm{m}(\mathrm{J})$. (A) short, straight chain, broad girdle view; (B, C) external and internal views of valves, areola array oriented towards marginal labiate process; (D) external opening of labiate process; (E) labiate process, internal view, 2-3 areolae away from valve margin; (F, G) open bands of cingulum with ligula and antiligula; $(\mathrm{H})$ external view of costate ocellus, hyaline ring not clear; (I) part of cingulum, notice the elongated pores of bands and the non-perforated area to the advalvar side (arrows); (J) areolae on valve face; (K) inside view of costate ocellus with a hyaline marginal ring. 

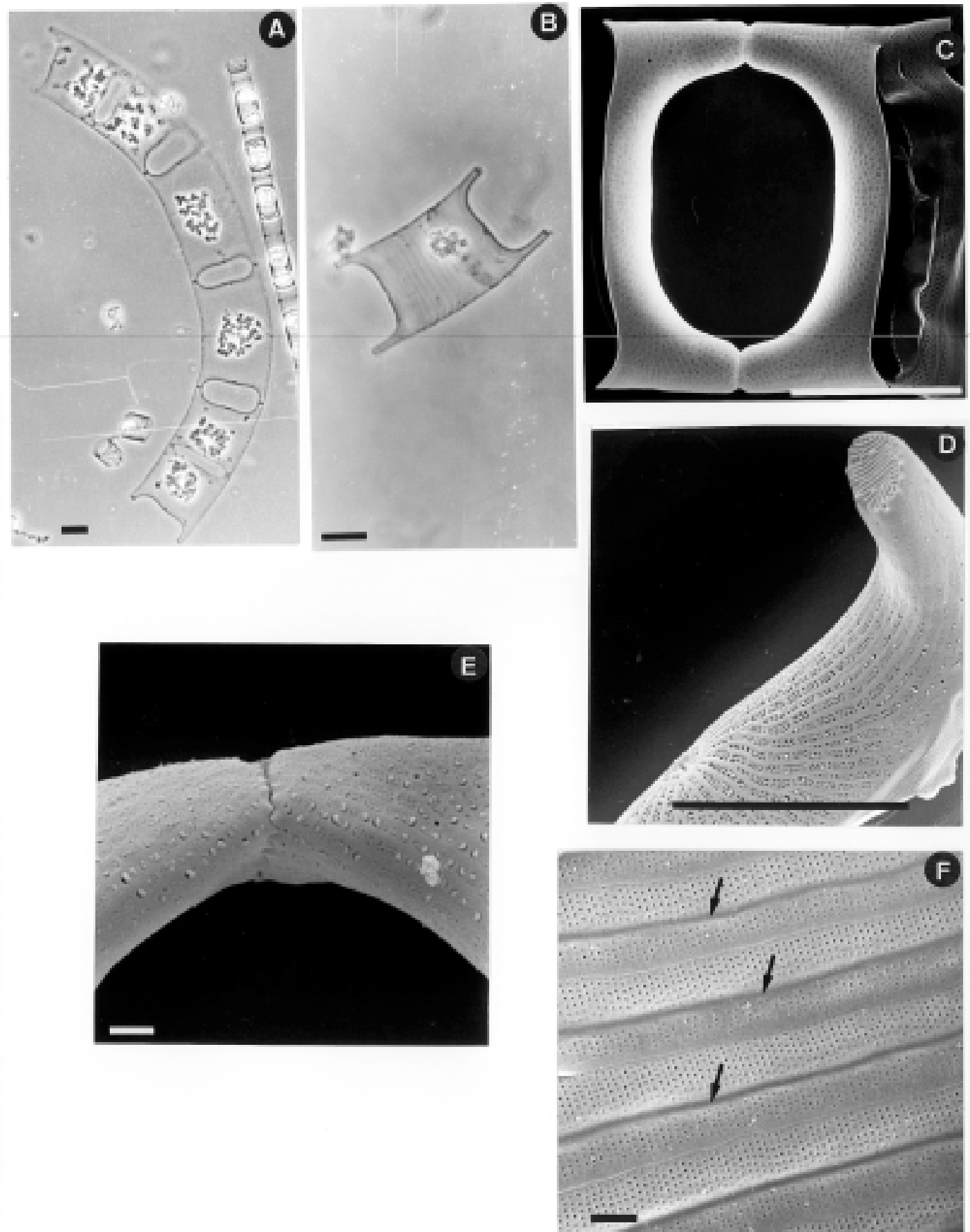

Fig. 3: Eucampia zodiacus Ehr. f. cylindrocornis Syvertsen. (A, B) ML, (C-F) MEB. Escala: $10 \mu \mathrm{m}$ (A-D), $1 \mu \mathrm{m}$ (E, F). (A) células curvadas en vista conectival ancha; (B) célula solitaria, note las largas elevaciones en los polos; (C) valvas típicas en vista conectival ancha; (D) abertura externa del proceso labiado central, orientación de las aréolas y costate ocellus oval con costillas paralelas en el centro pero curvadas en los extremos; (E) dientes marginales de los costate ocelli; (F) parte del cingulum, note un área no perforada en el centro de cada banda (flechas).

Eucampia zodiacus Ehr. f. cylindrocornis Syvertsen. (A, B) LM, (C-F) SEM. Scale: $10 \mu \mathrm{m}$ (A-D), $1 \mu \mathrm{m}(\mathrm{E}, \mathrm{F})$. (A) cells curved in broad girdle view; (B) single cell, note the long horns at the poles; (C) typical valves in broad girdle view; (D) external opening of central labiate process, areola array and oval costate ocellus with parallel ribs at the center but curved at the ends; (E) marginal teeth of costate ocelli; (F) part of girdle, note a non-perforated area at the center of each band (arrows). 
visto de pequeñas espinas o prolongaciones marginales (Fig. 3E), con costillas paralelas en la parte central y luego curvadas cerca de los extremos (Fig. 3D).

Distribución: este taxón, que no había sido señalado anteriormente para la costa chilena, se distribuye entre Arica $\left(18^{\circ} 28^{\prime} S\right)$ y la Bahía de Corral (3952’ S).

Eucampia zodiacus Ehrenberg f. recta Rivera, Avaria \& Cruces f. nov., Fig. 4.

Diferens forma zodiacus per colonias facie connectivali visae rectae et per minorem longitudinem valvarum $(18-39 \mu \mathrm{m})$.

Holotypus: Lamina DIAT-CONC 172.

Localidad tipo: Bahía de Concepción, Caleta El Morro, Talcahuano, 04.12.1965, M-71, Chile, DIAT-CONC M- 2199.

Material estudiado: DIAT-CONC M- 871, 1054, 2199, 2093, 2263, 2419, 2420, 2734, 3188, 3199.

Células cilíndricas, unidas en cadenas derechas en vista conectival ancha, pero levemente giradas respecto al eje pervalvar (Fig. 4A, 4B). Cingulum formado por bandas abiertas (Fig. 4H, 4I), 4-10 en cada teca, con líneas paralelas de poros redondeados o algo alargados (Fig. 4I, $4 \mathrm{~J}), 32-43$ en $10 \mu \mathrm{m}$; un área no perforada se presenta hacia el lado advalvar de cada banda (Fig. 4J). Valvas elípticas a lanceoladas (Fig. 4D), $18-39 \mu \mathrm{m}$ de largo, con sus elevaciones polares cortas $(2,5-6 \mu \mathrm{m})$ y cónicas en vista conectival ancha (Fig. 4A-4D, 4F, 4H), dejando aberturas elípticas a angular-elípticas entre dos células adyacentes (Fig. 4A, 4B, 4H). Aréolas de contorno cuadrangular (Fig. 4E, 4F, 4J), 1420 en $10 \mu \mathrm{m}$, dispuestas en líneas radiales a partir del proceso labiado que se ubica en una pequeña depresión en el centro de la valva (Fig. 4C, 4D); proceso labiado no prolongado hacia el exterior, pero bastante prominente hacia el interior (Fig. 4G). Costate ocelli ovalados a subcirculares, sin espinas marginales, provistos de costillas radiales que dejan una pequeña área central no perforada (Fig. 4D-4F). Difiere de la forma zodiacus por sus cadenas derechas en vista conectival ancha y por la menor longitud de las valvas.

Distribución: fue encontrada en muestras recolectadas entre Iquique (apróx. 20¹2'S) y la Bahía de Corral (39 $52^{\prime}$ 'S). Fue señalada como Climacodium biconcavum Cleve para la Bahía de Concepción por Rivera (1969).

Eucampia cornuta (Cleve) Grunow in Van Heurck Lám. 95bis, fig. 5 (1883), Fig 5.

Referencias: Syvertsen \& Hasle (1983), Hasle \& Syvertsen (1996).
Material estudiado: DIAT-CONC M- 1356, 2420, 2433, 3189, 3199. Células dos o más veces más largas que anchas, unidas en cadenas curvadas en vista conectival ancha (hasta cuatro células unidas fueron observadas, aunque la gran mayoría correspondió a células solitarias) (Fig. 5A-5C). Cada cingulum está formado por 8-11 bandas en el material analizado (Fig. 5B, 5C), cada una provista de líneas paralelas de poros de contorno más o menos cuadrangular (Fig. 5G, 5I, 5J), 44-51 en $10 \mu \mathrm{m}$; un área no perforada se sitúa un poco más cerca del lado advalvar de cada banda (Fig. 5I, 5J). Valvas elípticas, 13-29 $\mu \mathrm{m}$ de largo, con elevaciones largas y angostas $(11-18 \mu \mathrm{m})$ que dejan una gran abertura elíptica entre dos valvas adyacentes (Fig. 5A-5E). Aréolas rectangulares, dispuestas en líneas radiales a partir del proceso labiado central (Fig. 5E), 1115 en $10 \mu \mathrm{m}$ sobre la cara valvar pero más numerosas en las elevaciones valvares. Proceso labiado ubicado en una pequeña depresión de la valva, sin tubo externo (Fig. 5E), pero bastante prolongado hacia el lado interior (Fig. $5 \mathrm{G}, 5 \mathrm{H}$ ). Costate ocelli provistos de costillas paralelas, apicalmente orientadas, y terminando en el margen en estructuras a modo de espinas o dientes (Fig. 5E, 5F).

Distribución: había sido señalada desde los $18^{\circ} 28^{\prime}$ S hasta la Bahía de Concepción $-36^{\circ} 42^{\prime}$ S (Rivera 1983, Rivera et al. 1990). El presente estudio extiende su área de distribución hasta $\operatorname{los} 38^{\circ} 00^{\prime} \mathrm{S}$.

\section{DISCUSIÓN}

Sobre la base de las características de las cadenas, los dos géneros de la familia Hemiaulaceae presentes en aguas chilenas son fáciles de reconocer, incluso en muestras líquidas y utilizando microscopía fotónica. En Cerataulina estas son derechas, pero levemente giradas respecto al eje pervalvar, y las valvas presentan elevaciones muy cortas, provistas de una expansión aliforme, de modo que la distancia entre dos valvas adyacentes en un filamento es muy pequeña. Por el contrario, en el género Eucampia, las elevaciones valvares son mucho más largas, dejando siempre una gran abertura entre dos valvas adyacentes. Además, las cadenas pueden ser rectas o curvadas en vista conectival ancha.

Cerataulina pelagica, especie cosmopolita y el único taxón de este género encontrado hasta el presente en aguas chilenas, se diferencia de las otras dos especies válidamente descritas, $C$. dentata Hasle y C. bicornis (Ehrenberg) Hasle (Hasle \& Syvertsen 1996), por presentar el proceso labiado en posición central o subcentral, 



Fig. 4: Eucampia zodiacus Ehr. f. recta f. nov. (A) ML, (B-J) MEB. Escala: $10 \mu \mathrm{m}(\mathrm{A}-\mathrm{D}, \mathrm{H}), 1 \mu \mathrm{m}$ (E-G, I, J). (A, B) típicas cadenas derechas en vista conectival ancha; (C, D) proceso labiado central, orientación de las aréolas y costate ocellus; (E, F) costate ocelli con costillas radiales y pequeña área central, aréolas de contorno cuadrangular; $(\mathrm{G})$ vista interna del proceso labiado; $(\mathrm{H})$ típica célula en vista conectival ancha, note las cortas y anchas elevaciones valvares y las aberturas elípticas; (I, J) cingula: note los poros redondeados o alargados de las bandas y el área no perforada hacia el lado advalvar (flechas).

Eucampia zodiacus Ehr. f. recta f. nov. (A) LM, (B-J) SEM. Scale: $10 \mu \mathrm{m}$ (A-D, H), $1 \mu \mathrm{m}$ (E-G, I, J). (A, B) typical straight chains in broad girdle view; $(\mathrm{C}, \mathrm{D})$ central labiate process, areolae array and costate ocellus; (E, F) costate ocelli with radial ribs and small central area, areolae square in outline; $(\mathrm{G})$ internal view of labiate process; $(\mathrm{H})$ typical cell in broad girdle view, note short and broad horns at the poles and elliptical apertures; (I, J) cingula: note the round or elongated pores of the bands and the non-perforated area toward the advalvar side (arrows). 

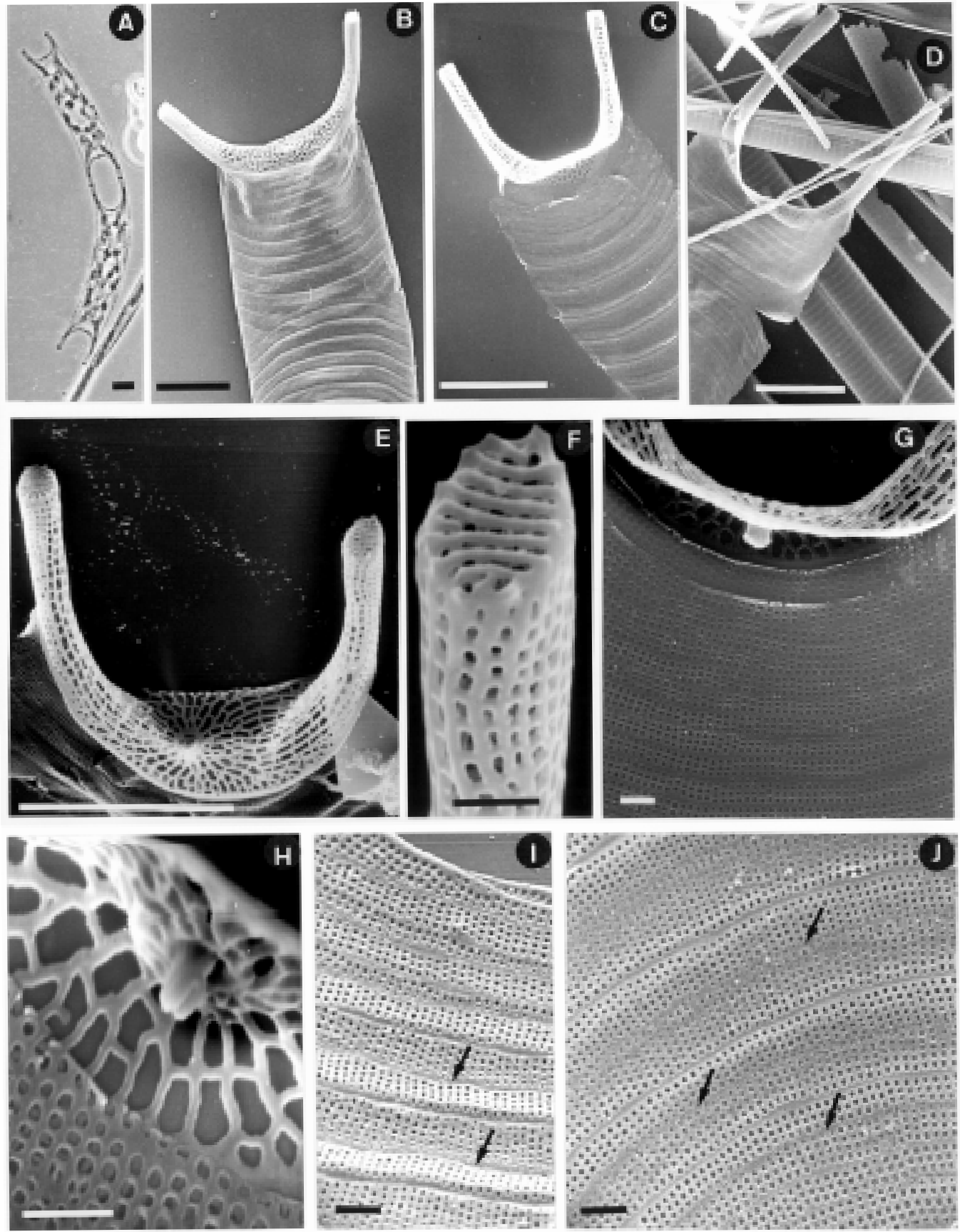

Fig. 5: Eucampia cornuta (Cleve) Grunow. (A) ML, (B-J) MEB. Escala: $10 \mu \mathrm{m}$ (A-E), $1 \mu \mathrm{m}$ (F-J). (A) típica cadena en vista conectival ancha, células con eje pervalvar y elevaciones valvares largas; (B-D) parte de células en vista conectival ancha mostrando las largas y angostas elevaciones valvares y las numerosas bandas del cingulum; (E) aréolas rectangulares orientadas hacia el proceso labiado central y largas elevaciones valvares con costate ocelli; (F) costate ocellus con costillas paralelas; $(\mathrm{G}, \mathrm{H})$ proceso labiado hacia el interior de la valva; (I, J) parte del cingulum con bandas perforadas por poros cuadrangulares, un área no perforada se ubica hacia el lado advalvar (flechas).

Eucampia cornuta (Cleve) Grunow. (A) LM, (B-J) SEM. Scale: $10 \mu \mathrm{m}$ (A-E), $1 \mu \mathrm{m}$ (F-J). (A) typical curved chain in broad girdle view, cells with long pervalvar axis and horns; (B-D) part of cells in broad girdle view showing long and narrow horns and numerous bands of the cingulum; (E) rectangular areolae oriented towards the central labiate process, and long horns with costate ocelli; (F) costate ocellus with parallel ribs; $(\mathrm{G}, \mathrm{H})$ labiate process on inside of valve; (I, J) parts of cingulum, arrows point to the non-perforated area toward the advalvar side. 
HEMIAULACEAE (BACILLARIOPHYCEAE) DE CHILE

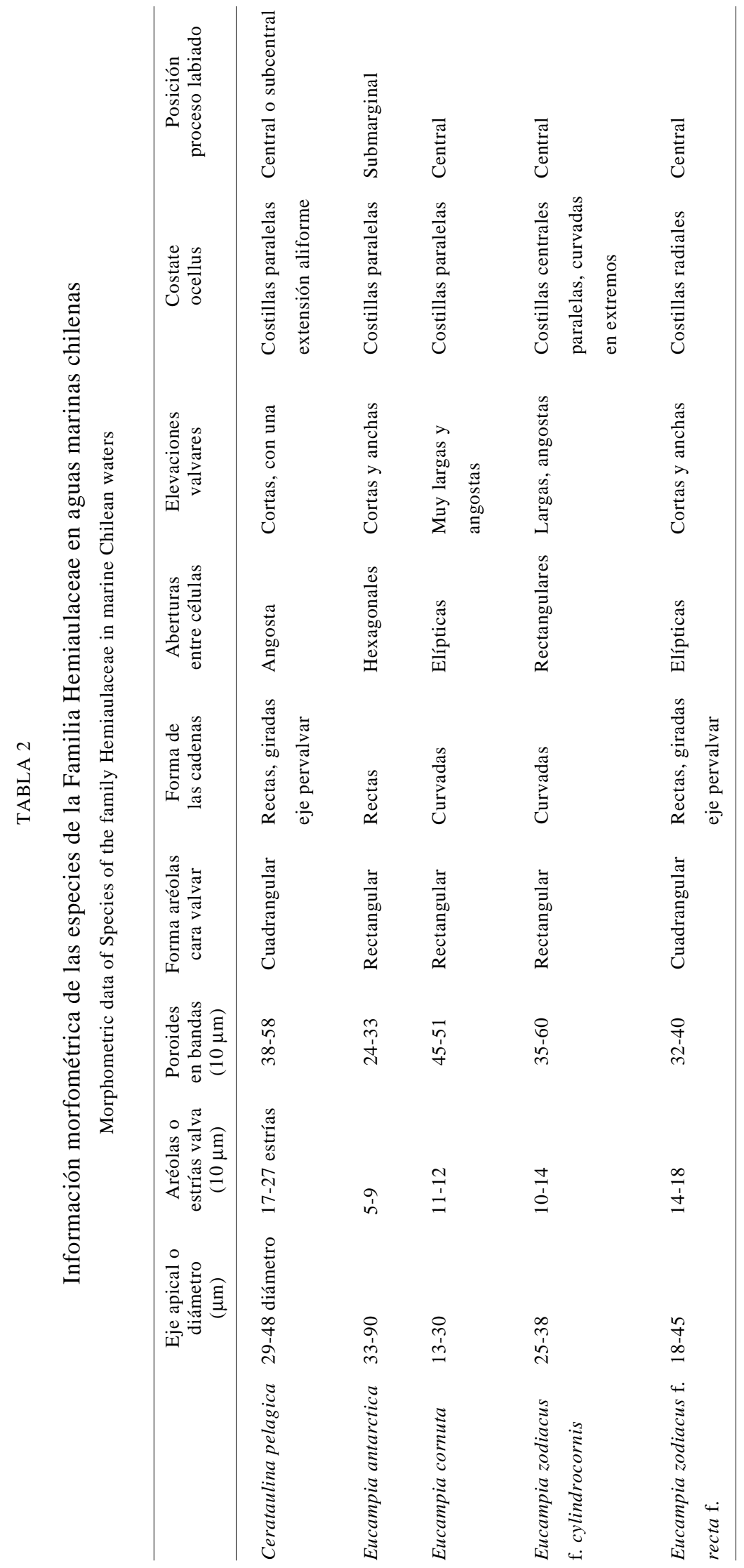


característica que también puede ser observada cuando se emplea microscopía fotónica. Otras diferencias relacionadas con la orientación de las estrías sobre la cara valvar, o inherentes a las elevaciones valvares, solamente pueden ser visualizadas con exactitud al emplearse técnicas de microscopía electrónica. La información morfométrica obtenida del material chileno coincide plenamente con la encontrada en la literatura para esta especie, con excepción del número de poros en las bandas que es algo menor que el señalado por Hasle \& Syvertsen (1980).

Eucampia antarctica es la única especie del género que presenta el proceso labiado en posición submarginal, característica también visible con microscopía fotónica. Además, la gran silicificación de sus células constituye otra gran diferencia con las restantes especies del género. Como el material estudiado de esta especie fue recolectado hace casi tres décadas, la gran mayoría de las células observadas no estaban unidas en cadenas; sin embargo, algunas cadenas rectas, formadas por dos a tres células, pudieron ser observadas incluso con microscopía electrónica. Este hecho nos hizo ver que nuestras células no correspondían plenamente con E. antarctica var. antarctica, que presenta cadenas curvadas en vista conectival ancha. En 1990 Fryxell \& Prasad describieron la variedad recta, caracterizada principalmente por sus colonias derechas en vista conectival ancha, pero algo arqueadas en vista conectival angosta, coincidiendo así plenamente con nuestro material. Otras características de nuestro material que corresponden bien con aquellas de la var. recta son el gran tamaño del eje apical (hasta $90 \mu \mathrm{m}$, y solamente $20-40 \mu \mathrm{m}$ en la var. antarctica), el mayor número de aréolas (rectangulares a angulares) en la cara valvar de las células vegetativas (hasta 9 en $10 \mu \mathrm{m}$, y 5-6 en $10 \mu \mathrm{m}$ en la var. antarctica), y la posición del proceso labiado situado a 2-3 aréolas del margen valvar. Sin embargo, características del costate ocellus presentes en cada una de las elevaciones valvares no parecen corresponder con aquellas presentes en las células vegetativas de la var. recta. Efectivamente, en esta última variedad el costate ocellus está rodeado de un notorio anillo hialino, no perforado, sin espinas, que no parece existir en algunas células de nuestro material (Fig. 2B, 2H) y su reconocimiento en vistas internas de otras células no es lo suficientemente claro (Fig. 2C, 2K). Sobre la base de lo anteriormente expuesto no podemos definir con precisión la variedad de E. antarctica presente en nuestro material, aunque pensamos que es más cercana a la var. recta. De igual forma, la escasa información morfológica entregada por
Meyer (1966) sobre el material recolectado en el Paso de Drake, y el no haber podido disponer de esas muestras para nuestro propio análisis, impiden precisar a qué variedad de E. antarcti$c a$ corresponde. No se encontraron esporas de reposo en el material estudiado de esta especie, aunque Hoban et al. (1980) y Syvertsen \& Hasle (1983) demostraron que tiene la capacidad de producirlas y describieron sus características morfológicas.

En este trabajo se señala por primera vez para las aguas chilenas a Eucampia zodiacus $\mathrm{f}$. cylindrocornis, taxón que se diferencia de la forma típica por el contorno y largo de las elevaciones valvares que son cilíndricas, más largas y delgadas, y por poseer costate ocelli con costillas paralelas en el centro pero curvadas en los extremos. La forma zodiacus también presenta cadenas curvadas en vista conectival ancha, pero sus elevaciones valvares son cortas y cónicas, y el costate ocellus lleva costillas radialmente orientadas (Syvertsen \& Hasle 1983). Hasta la fecha, E. zodiacus f. cylindrocornis fue confundida en Chile con la forma zodiacus (Rivera 1969), la cual no fue encontrada en el material analizado en este estudio. Sus características morfométricas coinciden plenamente con la descripción entregada por Syvertsen para este taxón, con excepción de que a veces existe un mayor número de poros en las bandas del cingulum (35-60 en 10 $\mu \mathrm{m}$ en lugar de 32-38).

Eucampia zodiacus f. recta $\mathrm{f}$. nov. se distingue de las restantes formas de la especie por sus cadenas rectas, largas, levemente giradas con respecto al eje principal de las mismas, y por el contorno cuadrangular de las aréolas presentes sobre la cara valvar. Cada costate ocellus lleva costillas radialmente orientadas, como los de la f. zodiacus, con la diferencia que el área central del ocellus es de un tamaño notoriamente menor. Además, en la f. zodiacus, el tamaño del eje apical de las células puede alcanzar hasta los $80 \mu \mathrm{m}$, no sobrepasando los $45 \mu \mathrm{m}$ en la f. recta f. nov. Aunque Eucampia groenlandica Cleve también puede presentarse formando cadenas rectas, y a veces giradas helicoidalmente, se diferencia de E. zodiacus f. recta f. nov. por presentar costate ocelli con costillas ordenadas en forma paralela, y por sus células muy poco silicificadas. Por otra parte, se sabe actualmente que la distribución geográfica de E. groenlandica está limitada a las aguas frías del Hemisferio Norte (Hasle \& Syvertsen 1996).

Eucampia cornuta es fácil de reconocer por sus largas y angostas elevaciones valvares, por la gran longitud del eje pervalvar respecto al eje apical, y por sus cadenas curvadas en vista 
conectival ancha. El número de poros encontrados en las bandas del material chileno (44-51 en $10 \mu \mathrm{m})$ es superior al señalado por Syvertsen \& Hasle (1983) para esta especie (26-31). En esa misma publicación, Syvertsen \& Hasle (1983) señalan como característica distintiva de E. cornuta, el presentar una costilla muy silicificada en el lado advalvar de cada una de sus bandas. En el material chileno de E. cornuta también fue posible observar esta característica, la que no fue exclusiva solo de esta especie, sino que se presentó también en todos los taxa del género, incluso en Cerataulina.

Sobre la base de los resultados obtenidos en el presente estudio, que incluyó la mayor parte del material previamente analizado por otros investigadores nacionales, podemos afirmar que son erróneas las citas de los géneros $\mathrm{He}$ miaulus Heiberg (H. sinensis Greville, H. membranaceus Cleve) y Climacodium Grunow ( $C$. biconcavum Cleve) para las aguas chilenas (Rivera 1969, Alvial \& Avaria 1982, Avaria \& Muñoz 1982, Rodríguez \& Escribano 1996). En Hemiaulus las elevaciones valvares son largas, puntiagudas, y carecen de costate ocelli; en $\mathrm{Cli}$ macodium el eje pervalvar es muy corto y las aberturas entre las células de una cadena son de gran tamaño. Ninguna de estas características fue observada en el material chileno analizado, y en las muestras donde estos géneros fueron señalados por otros investigadores nacionales encontramos solamente a Eucampia zodiacus f. recta f. nov. y a E. cornuta. Por otra parte, en las publicaciones de Avaria \& Muñoz (1991) y Muñoz et al. (1991), cuyo material no fue posible analizar en el presente trabajo, el autor S. Avaria (comunicación personal) está de acuerdo que las características morfológicas de las especies determinadas como $C$. biconcavum y $H$. sinensis corresponden plenamente con aquellas presentes en E. zodiacus f. recta f. nov. Indudablemente, la no utilización de técnicas de microscopía electrónica en esos trabajos, impidió la correcta visualización de las elevaciones valvares y las características relacionadas con el costate ocellus.

\section{AGRADECIMIENTOS}

Los autores agradecen a dos revisores anónimos por sus valiosos comentarios al manuscrito. Agradecemos también a los señores Raúl Alarcón, Hugo Pacheco y Julio Pugin del Laboratorio de Microscopía Electrónica de la Universidad de Concepción, por su ayuda en la preparación de las muestras y uso de los microscopios electrónicos. Esta investigación fue financiada parcialmente por la Dirección de Investigación Científica de la Universidad de Concepción.

\section{LITERATURA CITADA}

ALVIAL A \& S AVARIA (1982) Proliferación de primavera del fitoplancton en la Bahía de Valparaíso. II. Dinámica de las comunidades. Revista de Biología Marina de Valparaíso (Chile) 18: 1-56.

AVARIA S \& P MUNOZ (1982) Producción actual, biomasa y composición específica del fitoplancton de la Bahía de Valparaíso en 1979. Revista de Biología Marina de Valparaíso (Chile) 18: 129-157.

AVARIA S \& P MUÑOZ (1991) Caracterización del fitoplancton de un área de desove de merluza frente a la costa central de Chile. Revista de Biología Marina de Valparaíso (Chile) 26: 161-190.

AVARIA S, D CASSIS, P MUÑOZ \& P VERA (1997) Distribución del microfitoplancton marino en aguas interiores del sur de Chile en octubre de 1995 (Crucero CIMAR-FIORDO 1). Ciencia y Tecnología del Mar (Chile) 20: 107-123

FRYXELL G (1989) Marine phytoplankton at the Weddell Sea ice edge: seasonal changes at the specific level. Polar Biology 10: 1-18.

FRYXELL G \& AKSK PRASAD (1990) Eucampia antarctica var recta (Mangin) stat. nov. (Biddulphiaceae, Bacillariophyceae): life stages at the Weddel Sea ice edge. Phycologia 29: 27-38.

FRYXELL G, AKSK PRASAD \& PA FRYXELL (1989) Eucampia antarctica (Castracane) Mangin (Bacillariophyta): complex nomenclatural and taxonomic history. Taxon 38: 638-640.

HASLE G \& G FRYXELL (1970) Diatoms: cleaning and mounting for light and electron microscopy. Transactions of the American Microscopy Society 89: 469-474.

HASLE G \& E SYVERTSEN (1980) The diatom genus Cerataulina: morphology and taxonomy. Bacillaria 3: 79-114

HASLE G \& E SYVERTSEN (1996) Marine diatom. In: Tomas C (ed) Identifying marine diatoms and dinoflagellates: 5-385. Academic Press. Inc., New York, New York, USA.

HOBAN MA, GA FRYXELL \& KR BUCK (1980) Biddulphioid diatoms: resting spores in Antarctic Eucampia and Odontella. Journal of Phycology 16: 591-602.

MEYER RM (1966) Contribución al estudio del fitoplancton del Paso de Drake (Recolectado durante el verano 1960-61). Cuaderno Ciencias del Mar, Universidad Católica de Valparaíso (Chile) 1: 39-82.

MUÑOZ P, S AVARIA \& M FARÍAS (1991) Uso de información satélite en el estudio de un nuevo fenómeno de marea roja en la Bahía de Valparaíso Chile. Revista de Biología Marina de Valparaíso (Chile) 26: 415-435.

RIVERA P (1969) Sinopsis de las diatomeas de la Bahía de Concepción, Chile. Gayana Botánica (Chile) 18: 3-111.

RIVERA P (1981) Beiträge zur Taxonomie und Verbreitung der Gattung Thalassiosira Cleve (Bacillariophyceae) in den Küstengewässern Chiles. Bibliotheca Phycologica, Band 56: 1-369.

RIVERA P (1983) A guide for references and distribution for the Class Bacillariophyceae in Chile between $18^{\circ} 28^{\prime} \mathrm{S}$ and $58^{\circ} \mathrm{S}$. Bibliotheca Diatomologica, Band 3. J. Kramer, Vaduz. 386 pp.

RIVERA P (1985) Las especies del género Nitzschia Hassall, Sección Pseudonitzschia (Bacillariophyceae), en las 
aguas marinas chilenas. Gayana Botánica (Chile) 42: 9-38.

RIVERA P \& P KOCH (1984) Contributions to the Diatom Flora of Chile II. En: Mann DG (ed) Proceedings of the Seventh International Diatom Symposium, Philadelphia, USA: 279-298. Koeltz Scientific Books, Koenigstein, Germany.

RIVERA P, M GEBAUER \& HL BARRALES (1990) A guide for references and distribution for the Class Bacillariophyceae in Chile between $18^{\circ} 28^{\prime} \mathrm{S}$ and $58^{\circ}$ S. Part II. Data from 1982 to 1988. Gayana Botánica (Chile) 46: 155-198.

RIVERA P, L HERRERA \& H BARRALES (1996) Report of two species of Thalassiosira (Bacillariophyceae): $T$. rotula Meunier and $T$. anguste-lineata (A. Schmidt) Fryxell et Hasle, as new to northern Chile. Cryptogamie Algologie 17: 123-130.

RIVERA P, F CRUCES \& A CLEMENT (2002) Leptocylindrus minimus Gran (Bacillariophyceae): morfología y distribución en Chile. Gayana Botánica (Chile) 59: 7-11.

RIVERA P, F CRUCES \& I VILA (2003) Primera cita de Stephanodiscus agassizensis Hakansson \& Kling (Bacillariophyceae) en Chile. Gayana Botánica (Chile) 59: 79-86
RODRÍGUEZ L \& R ESCRIBANO (1996) Bahía Antofagasta y Bahía de Mejillones del Sur: observaciones de la temperatura, penetración de la luz, biomasa y composición fitoplanctónica. Estudios Oceanológicos (Chile) 15: 75-85.

ROSS R, PA SIMS \& GR HASLE (1977) Observations on some species of the Hemiauloideae. Nova Hedwigia Beihefte 54: 179-213.

ROSS R, EJ COX, NI KARAYEVA, DG MANN, TTB PADDOCK, R SIMONSEN \& PA SIMS (1979) An amended terminology for the siliceous components of the diatom cell. Nova Hedwigia Beihefte 64: 513-533.

SYVERTSEN E \& G HASLE (1983) The diatoms genus Eucampia: morphology and taxonomy. Bacillaria 6: 169-210.

TAKANO H (1990) Diatoms. In: Fukuyo Y, H Takano, M Chihara \& K Matsouka (eds) Red tide organism in Japan- an illustrated taxonomic guide: 162-331. Uchida Rokakuho, Tokyo, Japan.

VERA P, S AVARIA \& P MUÑOZ (1996) Composición y distribución del fitoplancton de los fiordos adyacentes a Campos de Hielo Sur y su relación con algunos parámetros oceanográficos. Ciencia y Tecnología del Mar (Chile) 19: 73-92.

Editor Asociado: Juan Correa

Recibido el 25 de enero de 2002; aceptado el 18 de agosto de 2003

ANEXO 1

Clave para la determinación de las especies de Eucampia Ehrenberg

Identification key to species of Eucampia Ehrenberg

1a. Proceso labiado submarginal E. antarctica

1b. Proceso labiado central 2

2a. Células derechas en vista conectival ancha, formando cadenas levemente giradas con respecto al eje pervalvar; elevaciones valvares cortas (hasta $6 \mu \mathrm{m}$ ), cónicas E. zodiacus f recta, f nov

2b. Células curvadas en vista conectival ancha; elevaciones valvares más largas (sobre $7 \mu \mathrm{m}$ ) 3

3a. Costate ocellus con costillas paralelas; elevaciones valvares largas (11-18 $\mu \mathrm{m}) \mathrm{y}$ angostas E. cornuta

3b. Costate ocellus con costillas paralelas en el centro y curvadas cerca de los extremos; elevaciones valvares más cortas $(7-9 \mu \mathrm{m})$, subcilíndricas

E. zodiacus f. cylindrocornis 